\title{
Obtenção e modelagem das isotermas de dessorção e do calor isostérico para sementes de arroz em casca
}

\author{
Rough rice seed desorption isotherms and isosteric heat
}

\author{
Daniel Emanuel Cabral de OLIVEIRA'; Osvaldo RESENDE ${ }^{2}$; Rafael CÂNDIDO CAMPOS ${ }^{3}$; \\ Juliana Rodrigues DONADON
}

${ }^{1}$ Autor para correspondência, Mestre em Ciências Agrárias, Instituto Federal de Educação Ciência e Tecnologia Goiano - Câmpus Rio Verde - GO, Brasil, Laboratório de Pós-colheita de Produtos Vegetais. Rod. Sul Goiana, Km 01- Cx. Postal: 66 - CEP 75.901-970, Fones: (64) 3620-5641. Fax: (64) 3620-5640. oliveira.d.e.c@gmail.com ${ }^{2} \mathrm{Dr}$, Engenheiro Agrícola, Instituto Federal de Educação Ciência e Tecnologia Goiano - Câmpus Rio Verde GO, Brasil, osvresende@yahoo.com.br

${ }^{3}$ Graduandoo em Agronomia, Instituto Federal de Educação Ciência e Tecnologia Goiano - Câmpus Rio Verde GO, Brasil, rcandido30@gmail.com

${ }^{4} \mathrm{Dr}^{\mathrm{a}} \mathrm{e}$ em Agronomia, Universidade Federal do Mato Grosso do Sul - MS, Brasil, julianadonadon@yahoo.com.br

Recebido em: 14-04-2013; Aceito em: 14-05-2014

\begin{abstract}
Resumo
O conhecimento do equilíbrio higroscópico das sementes de arroz em casca permite manejar adequadamente o produto visando à manutenção de seu teor de água nos níveis recomendados para um armazenamento seguro. Já o calor isostérico integral de dessorção fornece informações teóricas a respeito da energia requerida durante o processo de secagem. Desta forma, objetivou-se determinar as isotermas de dessorção de água de sementes de arroz em casca da cultivar BRS Sertanejo nas temperaturas de $10 ; 20 ; 30$ e $40^{\circ} \mathrm{C}$ para atividades de água variando entre 0,10 e 0,71 , ajustando diferentes modelos matemáticos aos dados experimentais, e selecionando aquele que melhor representa o fenômeno. Para a obtenção do equilíbrio higroscópico, foi utilizado o método estático indireto, utilizando o equipamento Hygropalm Model Aw 1. Conclui-se que os modelos Chung-pfost, GAB, Sabbah, Cavalcanti Mata, Henderson Modificado, Henderson, Oswin e GAB modificado representam, adequadamente, a higroscopicidade das sementes de arroz em casca da cultivar BRS Sertanejo, sendo o tradicional modelo de Henderson selecionado para representar as isotermas de dessorção do produto. Verificou-se ainda que, quanto maior a temperatura, maiores são os valores de atividade de água para um mesmo teor de água de equilíbrio. O calor isostérico aumenta com a diminuição do teor de água. Os valores de calor isostérico variam de $2.572,45$ a $2.514,26 \mathrm{~kJ} \mathrm{~kg}^{-1}$, para teores de água de 3,2 a 13,8 (\% b.s.).
\end{abstract}

Palavras-chave adicionais: higroscopicidade; Oryza sativa; teor de água de equilíbrio.

\begin{abstract}
Knowing the hygroscopic equilibrium of rice seeds permits the adequate management of the product to keep its moisture content at the recommended levels for a safe storage. On the other hand, the integral isosteric heat provides theoretical information concerning the required energy during the drying process. So, the objective of this study was to determine rough rice (cultivar 'BRS Sertanejo') seed water desorption isotherms at the temperatures of $10,20,30$, and $40{ }^{\circ} \mathrm{C}$ for the water activities between 0.10 and 0.71 so as to adjust different mathematical models to the experimental data and so to select the one best representing the phenomenon. To get the hygroscopic equilibrium, the indirect static method was used with the help of the equipment Hygropalm Model Aw 1. The conclusion was that the Chung-pfost, GAB, Sabbah, Cavalcanti Mata, Henderson modified, Henderson, Oswin, and GAB modified models represent adequately the rough rice seed hygroscopicity. The Henderson model was the chosen one to represent the desorption isotherms of the product. It was also verified that, for the same equilibrium water content, the higher the temperature, the higher the values of water activity. Isosteric heat increases with water content reduction. The values of isosteric heat vary from 2572.45 to $2514.26 \mathrm{~kJ} \mathrm{~kg}^{-1}$ for water contents of 3.2 to $13.8 \%$, dry basis.
\end{abstract}

Additional keywords: equilibrium moisture content; hygroscopicity; Oryza sativa. 


\section{Introdução}

O arroz (Oryza sativa) é o cereal mais cultivado e consumido do mundo pelo homem em todos os continentes (ALONSO et al., 2005). O Brasil é o nono maior produtor mundial, colhendo aproximadamente 11,7 milhões de toneladas na safra de 2011/2012, oriunda de dois sistemas de cultivo: irrigado e de sequeiro. A região Sul concentra $50 \%$ da área cultivada e contribui com $78 \%$ da produção nacional, sendo o Rio Grande do Sul o Estado com maior produção de arroz, sendo o sistema, predominantemente, irrigado. Já a área plantada com arroz de sequeiro, também chamado de arroz de terras altas, está concentrada na região Centro-Oeste (Mato Grosso e Goiás), produzindo 744,8 mil toneladas na última safra (CONAB, 2012). Sendo assim, os estudos da higroscopicidade alinhada a processos de secagem e armazenamento são de grande importância para garantir a qualidade desse produto.

Todos os produtos agrícolas têm a capacidade de ceder ou absorver água do ambiente, tendendo a manter constantemente uma relação de equilíbrio entre seu teor de água e as condições do ar ambiente. O teor de água de equilíbrio é alcançado quando a pressão parcial de vapor de água no produto se iguala à do ar que o envolve (CORRÊA et al., 2005).

O equilíbrio higroscópico ocorre quando a pressão de vapor da água presente no produto e a pressão de vapor de água da atmosfera são iguais (ARAÚJO et al., 2001). Essas trocas podem acontecer por meio do ganho ou da perda de água, fenômenos conhecidos por adsorção e dessorção. A afinidade existente entre a água e os outros componentes (gordura, amido, açúcar, proteínas, etc.) de um produto define sua higroscopicidade (BROOKER et al., 1992).

A isoterma é uma curva que descreve, em um teor de água específico, a relação de equilíbrio de uma quantidade de água sorvida por componentes do material biológico e a pressão de vapor ou umidade relativa, a uma dada temperatura (PARK et al., 2001).

De acordo com RESENDE et al. (2006), a partir do estudo de uma isoterma, pode-se manejar adequadamente o produto visando à manutenção de seu teor de água até níveis recomendados para um armazenamento seguro, sendo importante para definir as condições mais adequadas para o armazenamento do arroz em casca nas diversas regiões do País, levando-se em consideração os dados de temperatura e umidade relativa.

Durante o processo de secagem, com ar ambiente, as condições de temperatura e umidade relativa não são constantes, o que torna a determinação das isotermas de equilíbrio indispensável à avaliação da interação do sólido com
- vapor d'água presente no ar de secagem (LEHN \& PINTO, 2004).

A determinação das isotermas ou curvas de equilíbrio higroscópico de sorção de um material pode ser expressa por equações matemáticas, que descrevem, por meio de diferentes modelos, a relação de dependência entre o teor de água de equilíbrio, a temperatura e a umidade relativa do ar (CORRÊA et al., 2005).

A utilização de equações matemáticas para estimar o teor de água de equilíbrio higroscópico apresenta a vantagem de predição de valores de atividade de água do produto, em condições ambientais de difícil determinação experimental (RESENDE et al., 2006).

RESENDE et al. (2006), ao estudarem as isotermas de sorção dos grãos de arroz em casca da cultivar Urucuia, para as condições de temperaturas de 25; $35 ; 45$ e $55^{\circ} \mathrm{C}$ e umidade relativa do ar entre 30 e $85^{\circ} \mathrm{C}$, ajustaram diferentes modelos matemáticos aos dados experimentais e concluíram que as isotermas apresentam-se do tipo II, típico aos produtos agrícolas, e que o modelo de Chung-Pfost foi o que melhor representou a higroscopicidade da cultivar estudada.

Ao determinarem as isotermas de adsorção e dessorção de arroz-vermelho, variedade PB01, para as condições de temperaturas de 30; 50 e $70^{\circ} \mathrm{C}$ e umidades relativas entre 11 e $97 \%$, usando o método estático, RODOVALHO et al. (2009) ajustaram diversos modelos e concluíram que, para a estimativa do equilíbrio higroscópico de arroz-vermelho, o modelo Chen Clayton foi o que melhor representou a higroscopicidade do produto para os processos de dessorção.

Considerando a importância do conhecimento da higroscopicidade dos produtos agrícolas, propôs-se, com o presente trabalho, determinar as isotermas de dessorção para diferentes condições de temperatura e atividades de água, e ajustar diferentes modelos matemáticos aos dados experimentais, selecionando aquele que melhor represente o fenômeno da dessorção do arroz em casca da cultivar BRS Sertanejo. Além disso, buscou-se, ainda, obter o calor isostérico integral de dessorção para esse produto.

\section{Material e métodos}

O experimento foi conduzido no Laboratório de Pós-Colheita de Produtos Vegetais do Instituto Federal de Educação, Ciência e Tecnologia Goiano-Câmpus Rio Verde. Foram utilizadas sementes de arroz em casca da cultivar BRS Sertanejo colhidas no mês de novembro de 2012, sendo o teor de água inicial das sementes de $13,6 \pm 0,3 \%$ base seca (b.s.).

Além do teor de água inicial, as sementes foram submetidas à secagem em estufa com ventilação de ar forçada a $50^{\circ} \mathrm{C}$, para se obter os 
demais teores de água de 12,9; 11,0; 7,6 e 3,5 (\% b.s.), determinados em estufa a $105 \pm 1{ }^{\circ} \mathrm{C}$, durante 24 horas, em duas repetições (BRASIL, 2009).

Para a obtenção dos dados de dessorção das sementes de arroz em casca, utilizou-se do método estático indireto, sendo a atividade de água $\left(\mathrm{a}_{\mathrm{w}}\right)$ determinada por meio do equipamento
Hygropalm Model Aw 1. Para o controle da temperatura, utilizou-se de uma câmara tipo B.O.D. regulada a $10 ; 20 ; 30$ e $40^{\circ} \mathrm{C}$.

Aos dados experimentais foram ajustados modelos matemáticos frequentemente utilizados para representação da higroscopicidade de produtos agrícolas (Tabela 1).

Tabela 1. Modelos matemáticos utilizados para predizer a higroscopicidade de produtos vegetais. Mathematical models used to predict the hygroscopicity of plant products.

\begin{tabular}{|c|c|c|}
\hline Designação do modelo & Modelo & \\
\hline $\mathrm{Xe}=\mathrm{a}-\mathrm{b} \cdot \ln \left[-(\mathrm{T}+\mathrm{c}) \cdot \ln \left(\mathrm{a}_{\mathrm{w}}\right)\right]$ & Chung-Pfost & (1) \\
\hline $\mathrm{Xe}=\exp \left[\mathrm{a}-(\mathrm{b} \cdot \mathrm{T})+\left(\mathrm{c} \cdot \mathrm{a}_{\mathrm{w}}\right)\right]$ & Copace & (2) \\
\hline$X e=\left(a \cdot b \cdot c \cdot a_{w}\right) /\left[\left(1-c \cdot a_{w}\right) \cdot\left(1-c \cdot a_{w}+b \cdot c \cdot a_{w}\right)\right]$ & GAB & (3) \\
\hline$X e=\left[\exp (a-b \cdot T) /-\ln \left(a_{w}\right)\right]^{1 / c}$ & Halsey Modificado & (4) \\
\hline$X e=a \cdot\left(a_{w}{ }^{b / T^{c}}\right)$ & Sabbah & (5) \\
\hline$X e=\exp \left\{a-(b \cdot T)+\left[c \cdot \exp \left(a_{w}\right)\right]\right\}$ & Sigma Copace & (6) \\
\hline$X e=\left[\ln (1-a w) /\left(a \cdot\left(T^{b}\right)\right)\right]^{1 / c}$ & Cavalcanti Mata & (7) \\
\hline$X e=\left[\ln \left(1-a_{w}\right) /(-a \cdot(T+b))\right]^{1 / c}$ & Henderson Modificado & (8) \\
\hline $\mathrm{Xe}=\left[\ln \left(1-\mathrm{a}_{\mathrm{w}}\right) /(-\mathrm{a} \cdot(\mathrm{T}+273,16))\right]^{1 / \mathrm{c}}$ & Henderson & (9) \\
\hline$X e=\left\{1 /\left[\left(1-a_{w}\right) \cdot(1 / a \cdot b+((a-1) / a \cdot b))\right]\right\}$ & BET & $(10)$ \\
\hline $\mathrm{Xe}=(\mathrm{a}+\mathrm{b} \cdot \mathrm{T}) /[(1-\mathrm{aw}) / \mathrm{aw}]^{1 / c}$ & Oswin & (11) \\
\hline$\left(\frac{c}{T}\right)$ & \multirow{2}{*}{ GAB Modificado } & \multirow{2}{*}{$(12)$} \\
\hline$\left(1-b \cdot a_{w}+\left(\frac{c}{T}\right) \cdot b \cdot a_{w}\right) \cdot\left(1-b \cdot a_{w}\right)$ & & \\
\hline
\end{tabular}
modelo.

Para o ajuste dos modelos matemáticos, foi realizada a análise de regressão não linear, pelo método Gauss Newton. Para verificar o grau de ajuste de cada modelo, foi considerada a significância do coeficiente de regressão pelo teste $t$, a magnitude do coeficiente de determinação $\left(R^{2}\right)$, os valores do erro médio relativo $(P)$, erro médio estimado (SE) e do teste de Qui-quadrado $\left(\chi^{2}\right)$. Considerou-se o valor do erro médio relativo inferior a $10 \%$ como um dos critérios para a seleção dos modelos, de acordo com MOHAPATRA \& RAO (2005).

$$
\begin{aligned}
& P=\frac{100}{N} \sum \frac{|Y-\hat{Y}|}{Y} \\
& S E=\sqrt{\frac{\sum(Y-\hat{Y})^{2}}{G L R}}
\end{aligned}
$$

$$
\chi^{2}=\frac{\sum(Y-Y)^{2}}{G L R}
$$

em que: $\mathrm{Y}$ : valor experimental; $\hat{\mathrm{Y}}$ : valor estimado pelo modelo; N: número de observações experimentais, e GLR os graus de liberdade do modelo (número de observações experimentais menos o número de coeficientes do modelo).

O calor isostérico líquido de sorção, para cada teor de água de equilíbrio, foi obtido utilizando-se da equação de Clausius-Clayperon (IGLESIAS \& CHIRIFE, 1976):

$$
\frac{\partial \mathrm{ln}\left(\mathrm{a}_{\mathrm{w}}\right)}{\partial \mathrm{T}}=\frac{\Delta \mathrm{h}_{\mathrm{st}}}{R \mathrm{~T}_{\mathrm{a}}^{2}}
$$

em que, $a_{w}$ : atividade de água, decimal; $T_{a}$ : temperatura absoluta, $\mathrm{K} ; \Delta \mathrm{h}_{\mathrm{st}}$ : entalpia diferencial ou calor isostérico líquido de sorção, $\mathrm{kJ} \mathrm{kg}^{-1} ; \mathrm{R}$ : constante para o vapor d'água $\left(0,4619 \mathrm{~kJ} \mathrm{~kg}^{-1} \mathrm{~K}^{-1}\right)$. 
Integrando a Equação 16 e assumindo que o calor isostérico líquido de sorção é independente da temperatura, para cada teor de água de equilíbrio, esse índice foi obtido conforme a Equação 17 (WANG \& BRENNAN, 1991):

$\ln (\mathrm{aw})=-\left(\frac{\Delta \mathrm{h}_{\mathrm{st}}}{\mathrm{R}}\right) \frac{1}{\mathrm{Ta}}+\mathrm{C}$

em que, C: coeficiente do modelo.

Os valores de atividade de água, temperatura e teor de água de equilíbrio foram obtidos a partir das isotermas de dessorção das sementes de arroz, utilizando o modelo de melhor ajuste aos dados experimentais. $O$ calor isostérico integral de sorção foi obtido adicionando-se aos valores de calor isostérico líquido de sorção o valor do calor latente de vaporização da água livre, de acordo com a Equação 18. Já o valor do calor latente de vaporização da água livre $(\mathrm{L})$, em $\mathrm{kJ} \mathrm{kg}^{-1}$, necessário ao cálculo de $Q_{\mathrm{st}}$, foi obtido utilizando-se da Equação 19:
$\mathrm{Q}=\Delta \mathrm{h}_{\mathrm{st}}+\mathrm{L}=\mathrm{a} \cdot \exp \left(-\mathrm{b} \cdot \mathrm{X \textrm {e } ^ { * }}\right)+\mathrm{L}$

$\mathrm{L}=2502,2-2,39 \mathrm{~T}$

em que, $Q_{\mathrm{st}}$ : calor isostérico integral de sorção $\left(\mathrm{kJ} \mathrm{kg}^{-1}\right)$; L: calor latente de vaporização da água livre $\left(\mathrm{kJ} \mathrm{kg}^{-1}\right)$; $\mathrm{Xe}^{\star}$ : teor de água de equilíbrio, \% b.s; a e b são coeficientes do modelo.

\section{Resultados e discussão}

Os valores médios das atividades de água das sementes de arroz em casca obtidos para diversos teores de água e temperaturas de 10; 20; 30 e $40^{\circ} \mathrm{C}$ são apresentados na Tabela 2.

$\mathrm{Na}$ Tabela 2, verifica-se que existe uma relação entre temperatura, atividade de água e teor de água de equilíbrio para as sementes de arroz em casca, pois, para uma mesma temperatura, com o aumento da atividade de água, tem-se um aumento sensível do teor de água de equilíbrio. Observa-se que, com o aumento da temperatura, o teor de água de equilíbrio decresce. Costa et al. (2013), estudando a higroscopicidade de frutos de crambe, verificaram 0 mesmo comportamento.

Tabela 2 - Valores médios do teor de água de equilíbrio (\% b.s.) das sementes de arroz em casca da cultivar BRS Sertanejo, obtidos pelo processo de dessorção, em função da temperatura $\left({ }^{\circ} \mathrm{C}\right)$ e da atividade de água. Mean values of equilibrium moisture content of rough rice seeds of the cultivar 'BRS Sertanejo') resulting from the desorption process as influenced by temperature and water activity.

\begin{tabular}{|c|c|c|c|c|}
\hline \multirow{2}{*}{ Atividade de água } & \multicolumn{4}{|c|}{ Temperatura $\left({ }^{\circ} \mathrm{C}\right)$} \\
\hline & 10 & 20 & 30 & 40 \\
\hline 0,109 & 3,2 & - & - & - \\
\hline 0.125 & - & 3.6 & - & - \\
\hline 0,134 & - & - & 3,9 & - \\
\hline 0,143 & - & - & - & 3,4 \\
\hline 0,336 & 7,4 & - & - & - \\
\hline 0,343 & - & 7,6 & - & - \\
\hline 0,370 & - & - & 7,6 & - \\
\hline 0,391 & - & - & - & 7,7 \\
\hline 0,534 & 10,8 & - & - & - \\
\hline 0,540 & - & 11,2 & - & - \\
\hline 0,554 & - & - & 10,9 & - \\
\hline 0,586 & - & - & - & 11,1 \\
\hline 0,635 & 12,6 & - & - & - \\
\hline 0,641 & - & 13,0 & - & - \\
\hline 0,657 & - & - & 12,8 & \\
\hline 0,671 & 13,6 & - & - & - \\
\hline 0,672 & - & - & - & 13,0 \\
\hline 0,683 & - & 13,5 & - & - \\
\hline 0,701 & - & - & 13,3 & - \\
\hline 0,708 & - & - & - & 13,8 \\
\hline
\end{tabular}

Na Tabela 3, estão apresentados os coeficientes dos modelos de equilíbrio higroscópico, bem como o coeficiente de determinação $\left(R^{2}\right)$, os valores do erro médio relativo $(P)$, erro médio estimado $(\mathrm{SE})$ e do teste de Qui-quadrado $\left(\chi^{2}\right)$, para as sementes de arroz em casca, obtidos por dessorção, para diferentes condições de temperatura. 
Tabela 3 - Parâmetros dos modelos de equilíbrio higroscópico para as sementes de arroz em casca da cultivar BRS Sertanejo, com seus respectivos coeficientes de determinação $\left(R^{2}\right.$, \%), erros médios relativo $(P, \%)$ e estimado (SE, decimal), qui-quadrado $\left(\chi^{2}\right.$, decimal) e coeficientes dos modelos ajustados ( $\mathrm{a}, \mathrm{b}$ e $\mathrm{c}$ ). Parameters of models of equilibrium moisture content of rough rice cultivar ' $\mathrm{BRS}$ Sertanejo' with their respective coefficient of determination $\left(R^{2}, \%\right)$, mean relative error $(P, \%)$, chisquare, and adjusted model coefficients ( $a, b$, and $c)$.

\begin{tabular}{|c|c|c|c|c|c|c|c|}
\hline \multirow{2}{*}{ Modelos } & $\mathrm{R}^{2}$ & $P$ & SE & $\chi^{2}$ & \multicolumn{3}{|c|}{ Coeficientes } \\
\hline & \multicolumn{2}{|c|}{$(\%)$} & \multicolumn{2}{|c|}{ decimal } & a & $b$ & c \\
\hline 1 - Chung-pfost & 99,65 & 2,22 & 0,24 & 0,06 & $41,0699^{\star \star}$ & $5,9035^{\star \star}$ & $256,1039^{\star \star}$ \\
\hline 2 - Copace & 97,68 & 7,95 & 0,61 & 0,38 & $1,2636^{\star *}$ & $0,0023^{\mathrm{ns}}$ & $2,0707^{\star *}$ \\
\hline $3-\mathrm{GAB}$ & 99,30 & 3,41 & 0,34 & 0,11 & $9,7004^{* *}$ & $5,8423^{* *}$ & $0,6097^{* \star}$ \\
\hline 4 - Halsey Modificado & 96,97 & 8,91 & 0,70 & 0,49 & $3,0440^{* *}$ & $0,0040^{\text {ns }}$ & $1,4884^{* *}$ \\
\hline 5 - Sabbah & 99,41 & 3,56 & 0,31 & 0,10 & $20,2306^{\star *}$ & $0,8335^{\star \star}$ & $0,0328^{*}$ \\
\hline 6 - Sigma Copace & 95,49 & 11,26 & 0,86 & 0,73 & $0,1690^{\text {ns }}$ & $0,0026^{\mathrm{ns}}$ & $1,2756^{\star *}$ \\
\hline 7 - Cavalcanti Mata & 99,57 & 2,53 & 0,26 & 0,07 & $-0,0135^{\star *}$ & $0,0657^{\star *}$ & $1,6275^{\star \star}$ \\
\hline 8 - Henderson Modificado & 99,63 & 2,40 & 0,25 & 0,06 & $0,0001^{\star *}$ & $278,5836^{\star \star}$ & $1,6261^{* *}$ \\
\hline 9 - Henderson & 99,63 & 2,41 & 0,24 & 0,06 & $0,000056^{* *}$ & * $1,6261^{\star *}$ & - \\
\hline 10 - BET & 91,98 & 14,54 & 1,11 & 1,23 & $-102,237^{\text {ns }}$ & $0,227^{* *}$ & - \\
\hline 11 - Oswin & 98,97 & 4,63 & 0,41 & 0,17 & $10,1802^{* *}$ & $-0,0229^{* *}$ & $-2,1787^{* *}$ \\
\hline 12 - GAB modificado & 97,56 & 7,05 & 0,63 & 0,40 & $6,2244^{\star *}$ & $0,8395^{\star *}$ & $264,6145^{\star \star}$ \\
\hline
\end{tabular}

Avaliando-se a Tabela 3, nota-se que os modelos Chung-Pfost (1), GAB (3), Sabbah (5), Cavalcanti Mata (7), Henderson Modificado (8) e Henderson (9) apresentaram valores do coeficiente de determinação $\left(R^{2}\right)$ superiores a $99 \%$. Já o modelo de BET (10) apresentou o valor do coeficiente de determinação inferior a $95 \%$. Valor superior a $95 \%$ é desejável, pois indica uma representação satisfatória do fenômeno em estudo.

Com relação ao erro médio relativo $(P)$, nota-se que o modelo Chung-Pfost (1) apresentou o menor valor (2,22\%), já os modelos Sigma Copace (6) e BET (10) apresentaram valores superiores a $10 \%$ que, de acordo com MOHAPATRA \& RAO (2005), são valores não recomendados para a seleção de modelos.

O coeficiente de determinação $\left(R^{2}\right)$ e o erro médio relativo $(P)$ não são suficientes para avaliar o ajuste de um modelo de equilíbrio higroscópico (AVIARA et al., 2004). Desta forma, procedendo-se à avaliação do erro médio estimado (SE), verificou-se que o modelo de ChungPfost (1), Cavalcanti Mata (7), Henderson Modificado (8) e Henderson (9) apresentaram os menores valores. A capacidade de um modelo representar adequadamente um determinado processo físico é inversamente proporcional ao valor do erro médio estimado (DRAPER \& SMITH, 1981). Em relação ao teste de Qui-quadrado $\left(\chi^{2}\right)$, verificou-se que todos os modelos analisados se encontram no intervalo de confiança de $99 \%$.

Verifica-se ainda, na Tabela 3 , que à exceção dos modelos Copace (2), Halsey Modificado (4), Sigma Copace (6) e BET (10), todos os demais modelos apresentaram significância de seus coeficientes a $1 \%$ de significância, pelo teste de t.

Dessa forma, dentre os modelos avaliados, apenas os modelos Copace (2), Halsey Modificado (4), Sigma Copace (6) e BET (10) não são adequados para determinar a higroscopicidade das sementes de arroz em casca. Dentre os modelos que podem ser utilizados para determinar a higroscopicidade das sementes de arroz em casca, o modelo de Henderson apresentou elevado coeficiente de determinação e baixos valores dos erros médios relativo e estimado, bem como os coeficientes significativos a $1 \%$, sendo recomendado para predição do teor de água de equilíbrio higroscópico das sementes de arroz em casca pela tradição e simplicidade do modelo, indicado pela Equação 20. 
$\mathrm{Xe}=\left[\ln \left(1-\mathrm{a}_{\mathrm{w}}\right) /(-0,000056 * *(\mathrm{~T}+273,16))\right]^{1 / 1,626 * *}$

**Significativo a $1 \%$ de significância.

RESENDE et al. (2006) e GONELI et al. (2007), estudando o teor de água de equilíbrio de grãos de arroz em casca, verificaram que o modelo de Chung-Pfost foi o que melhor se ajustou aos dados experimentais, corroborando os resultados encontrados para as sementes de arroz em casca da cultivar BRS Sertanejo.

$\mathrm{Na}$ Figura 1, são apresentados os valores experimentais da atividade de água das sementes de arroz em casca, obtidas por dessorção, bem como suas isotermas estimadas por meio do modelo de Henderson.

Verifica-se que, com o aumento da temperatura, para um teor de água de equilíbrio constante, tem-se uma pequena elevação na atividade de água, seguindo a mesma tendência da maioria dos produtos agrícolas já estudados (MULET et al., 2002; TOLABA et al., 2004; AYRANCI \& DUMAN, 2005; RESENDE et al., 2006; RESENDE et al., 2006; CORRÊA et al., 2006).

As isotermas de dessorção obtidas para as sementes de arroz em casca da cultivar BRS Sertanejo apresentaram-se no formato sigmoidal, típico de diversos produtos agrícolas (AVIARA et al., 2004; CORRÊA et al., 2005; RESENDE et al., 2006; FURMANIAK et al., 2007; IGUAZ \& VÍRSEDA, 2007).

$\mathrm{Na}$ Figura 2, são apresentados os valores do calor isostérico integral de dessorção $\left(Q_{\mathrm{st}}\right)$, em função do teor de água de equilíbrio (\% b.s.).

$\mathrm{Na}$ Figura 2, verifica-se que o calor isostérico integral de dessorção das sementes de arroz é dependente do teor de água. A redução da quantidade de água proporcionou o aumento da energia necessária para a remoção de água das sementes. AVIARA \& AJIBOLA (2002) ressaltam que, em produtos com maior teor de água, a força de ligação entre as moléculas de água e a matéria seca diminui sensivelmente. Este comportamento é observado para diversos produtos vegetais como arroz em casca (RESENDE et al., 2006), milho-doce (OLIVEIRA et al., 2010), quiabo (GONELI et al., 2010) e caju-de-árvore-do-cerrado (CAETANO et al., 2012).

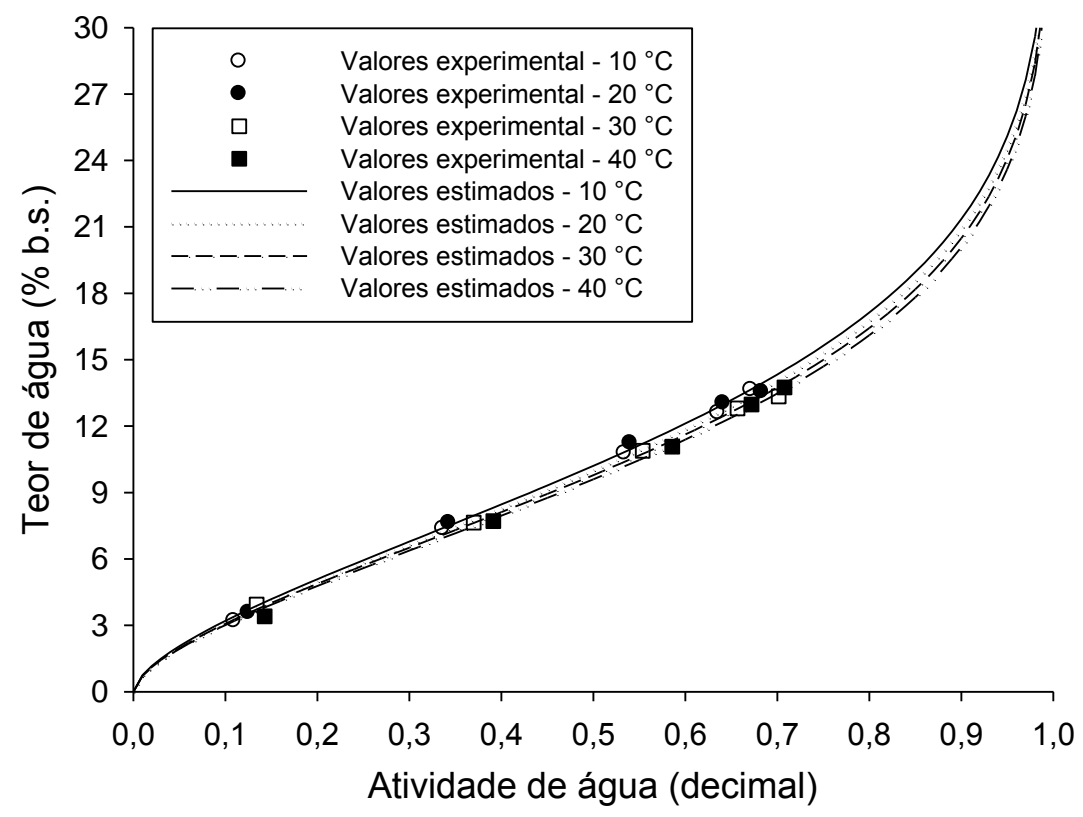

Figura 1 - Isotermas de dessorção de água para as sementes de arroz em casca da cultivar BRS Sertanejo, estimadas pelo modelo de Henderson, para diferentes condições de temperatura e atividade de água. Water desorption isotherms for rough rice seeds, cv. 'BRS Sertanejo', estimated by the Henderson model for different conditions of temperature and water activity.

Na Figura 2, verifica-se que o calor isostérico integral de dessorção das sementes de arroz é dependente do teor de água. A redução da quantidade de água proporcionou o aumento da energia necessária para a remoção de água das sementes. AVIARA \& AJIBOLA (2002) ressaltam que, em produtos com maior teor de água, a força de ligação entre as moléculas de água e a matéria seca diminui sensivelmente. Este comportamento é observado para diversos produtos vegetais como arroz em casca (RESENDE et al., 2006), milho-doce (OLIVEIRA et al., 2010), quiabo (GONELI et al., 2010) e caju-de-árvore-do-cerrado (CAETANO et al., 2012). 


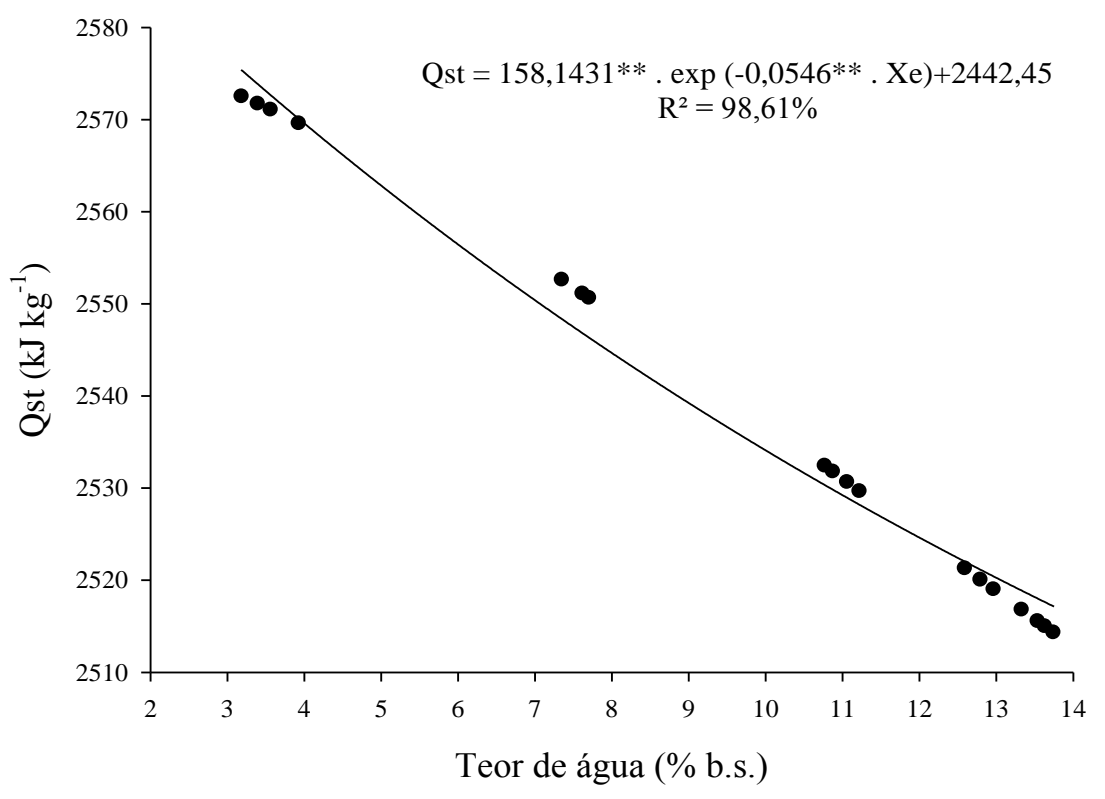

**Significativo a 1\% de significância.

Figura 2 - Calor isostérico integral de dessorção para as sementes de arroz em casca da cultivar BRS Sertanejo, em função do teor de água de equilíbrio. Desorption integral isosteric heat of rough rice, $\mathrm{CV}$. 'BRS Sertanejo', as influenced by the equilibrium water content.

Os valores de calor isostérico integral de dessorção, para as sementes de arroz em casca, na faixa de teor de água de 3,2 a 13,8 (\% b.s.), variaram de $2.572,45$ a $2.514,26 \mathrm{~kJ} \mathrm{~kg}^{-1}$. Já RESENDE et al. (2006) verificaram que o calor isostérico, para os grãos de arroz em casca, na faixa de teor de água de 12 a 19 (\% b.s.), varia de 3.860 a $2.712 \mathrm{~kJ} \mathrm{~kg}^{-1}$. As diferenças no valor de calor isostérico integral de dessorção podem estar relacionadas com o próprio produto e, segundo FERREIRA \& PENA (2003), também podem ser relacionadas com a obtenção dos valores de atividade de água, uma vez que os valores foram obtidos a partir de modelos matemáticos.

\section{Conclusões}

O teor de água de equilíbrio das sementes de arroz em casca é diretamente proporcional à atividade de água e decresce com o aumento da temperatura, para um mesmo valor de atividade de água.

Os modelos Chung-pfost, GAB, Sabbah, Cavalcanti Mata, Henderson Modificado, Henderson, Oswin e GAB modificado representam adequadamente a higroscopicidade das sementes de arroz em casca da cultivar BRS Sertanejo, sendo o tradicional modelo de Henderson selecionado para representar as isotermas de sorção do produto.

Com a redução do teor de água, ocorre aumento da energia necessária para a remoção de água do produto, sendo que os valores do calor isostérico integral de dessorção, para as sementes de arroz em casca da cultivar BRS Sertanejo, na faixa de teor de água de 3,2 a 13,8 (\% b.s.), variam de $2.572,45$ a $2.514,26 \mathrm{~kJ} \mathrm{~kg}^{-1}$.

\section{Referências}

ALONSO, S. A.; SANTOS, A. B.; GOMES, A. S. Cultivo do arroz irrigado no Brasil. Pelotas: Embrapa Clima Temperado, 2005. Versão Eletrônica disponível em: <http://www.embrapa.cnpaf.br>. Acesso em: 8 mar. 2013.

ARAÚJO, L. F.; CORRÊA, P. C.; SILVA, R. F. Comparação de modelos matemáticos para descrição das curvas de dessorção de sementes de milho-doce. Pesquisa Agropecuária Brasileira, Brasília, v.36, n.7, p.991-995, 2001.

AVIARA, N.A.; AJIBOLA, O. O. Thermodynamics of moisture sorption in melon seed and cassava. Journal of Food Engineering, Londres, v.55, n.2, p.107-113, 2002.

AVIARA, N. A.; AJIBOLA, O. O.; ONI, S. A. Sorption equilibrium and thermodynamic characteristics of soya bean. Biosystems Engineering, London, v.87, n.2, p.179-190, 2004.

AYRANCI, E.; DUMAN, O. Moisture sorption isotherms of cowpea (Vigna unguiculata L. Walp) and its protein isotate at 10,20 and $30^{\circ} \mathrm{C}$. Journal of Food Engineering, Londres, v.70, n.1, p.83-91, 2005. 
BRASIL. Ministério da Agricultura e Reforma Agrária. Secretaria Nacional de defesa Agropecuária. Regras ara análise de sementes. Brasília, DF, 2009. 398p.

BROOKER, D.B.; BAKKER-ARKEMA, F.W.; HALL, C.W. Drying and storage of grains and oilseeds. New York: van Nostrand Reinhold, 1992. 450p.

CAETANO, G. S.; SOUSA, K. A.; RESENDE, O.; SALES, J. F.; COSTA, L. M. Higroscopicidade de sementes de caju-de-árvore-do-cerrado. Pesquisa Agropecuária Tropical, Goiânia, v.42, n.4, p.437445, 2012.

CONAB. Companhia Nacional de Abastecimento. Acompanhamento de safra Brasileira: grãos, primeiro levantamento, outubro 2012 / Companhia Nacional de Abastecimento. Brasília, 2012.

CORRÊA, P. C.; RESENDE, O.; RIBEIRO, D. M. Isotermas de sorção das espigas de milho: obtenção e modelagem. Revista Brasileira de Milho e Sorgo, Sete Lagoas, v.4, n.1, p.126-134, 2005.

CORRÊA, P. C.; AFONSO JÚNIOR, P. C.; RIBEIRO, D. M.; SILVA, F. S. Equilíbrio higroscópico de milheto, alpiste e painço: Obtenção e modelagem. Revista Brasileira de Engenharia Agrícola e Ambiental, Campina Grande, v.10, n.1, p.162-167, 2006.

COSTA, L. M.; RESENDE, O.; OLIVEIRA, D. E. C. Isotermas de dessorção e calor isostérico dos frutos de crambe. Revista Brasileira de Engenharia Agrícola e Ambiental, Campina Grande, v.17, n.4, p.412-418, 2013.

DRAPER, N. R.; SMITH, H. Applied regression analysis. 3 ed. New York: John Wiley \& Sons, 1998. 712p.

FERREIRA, C.D.; PENA, R.S. Comportamento higroscópico da farinha de pupunha (Bactris gasipaes). Ciência e Tecnologia de Alimentos, Campinas, v.23, n.2, p.251-255, 2003.

FURMANIAK, S.; TERZYK, A. P.; GAUDEN, P. $A$. The general mechanism of water sorption on foodstuffs - Importance of the multitemperature fitting of data and the hierarchy of models. Journal of Food Engineering, Londres, v.82, n.4, p.528-535, 2007.

GONELI, A. L. D.; CORRÊA, P. C.; RESENDE, O.; NOGUEIRA, B. L.; BOTELHO, F. M. Modelagem matemática do equilíbrio higroscópico dos grãos de arroz em casca obtidos pelos métodos estáticos e dinâmico. Revista Brasileira de Armazenamento, Viçosa, MG, v 32, n.2, p.152160, 2007.

GONELI, A. L. D.; CORRÊA, P. C.; OLIVEIRA, G. H. H.; BOTELHO, F. M. Water desorption and thermodynamic properties of okra seeds.
Transactions of the ASABE, St. Joseph, v.53, n.1, p.191-197, 2010.

IGLESIAS, H.; CHIRIFE, J. Isosteric heats of water vapour sorption on dehydrated foods. Part II: hysteresis and heat of sorption comparison with BET theory. Lebensmittel Wissenschaft and Technologie, Zürich, v.9, n.1, p.123-127, 1976.

IGUAZ, A.; VÍRSEDA, P. Moisture desorption isotherms of rough rice at high temperatures. Journal of Food Engineering, Londres, v.79, n.3, p.794-802, 2007.

LEHN, D. N.; PINTO, L. A. A. Isotermas de equilíbrio e curvas de secagem para arroz em casca em silos de armazenagem. Engenharia na Agricultura, Viçosa, MG, v.12, n.3, p.177-191, 2004.

MOHAPATRA, D.; RAO, P. S. A thin layer drying model of parboiled wheat. Journal of Food Engineering, Londres, v.66, n.4, p.513-518, 2005.

MULET, A.; GARCA-PASCUAL, P.; SANJUAN, N.; GARCA-REVERTER, J. Equilibrium isotherms and isosteric heats of morel (Morchella esculenta). Journal of Food Engineering, Londres, v.53, n.1, p.75-81, 2002.

OLIVEIRA, G. H. H; CORRÊA, P. C.; ARAÚJO, E. F.; VALENTE, D. S. M.; BOTELHO, F. M. Desorption isotherms and thermodynamic properties of sweet corn cultivars (Zea mays L.). International Journal of Food Science and Technology, Malden, v.45, n.3, p.546-554, 2010.

PARK, K. J.; BIN, A.; BROD, F. P. R. Obtenção das isotermas de sorção e modelagem matemática para a pêra bartlett (Pyrus sp.) com e sem desidratação osmótica. Ciência e Tecnologia de Alimentos. Campinas, v.21, n.1, p.73-77, 2001.

RESENDE, O.; CORRÊA, P.C.; GONELI, A.L.D.; MARTINAZZO, A.P.; RIBEIRO, R.M. Isotermas e calor isostérico de dessorção do arroz em casca. Revista Brasileira de Armazenamento, Viçosa, MG, v.31, n.1, p.86-94, 2006.

RODOVALHO, R. S.; DEVILLA, I. A.; ASCHERI, D. P. R.; BASSINELLO, P. Z.; FERREIRA, D. A. Isotermas de sorção do arroz-vermelho em casca (Oriza sativa L.). Revista Brasileira de Armazenamento, Viçosa, MG, v.34, n.2, p.144$-152,2009$.

TOLABA, M. P.; PELTZER, M.; ENRIQUEZ, N.; POLLIO, M. L. Grain sorption equilibria of quinoa grains. Journal of Food Engineering, Londres, v.61, n.3, p.365-371, 2004.

WANG, N.; BRENNAN, J. G. Moisture sorption isotherm characteristics of potato at four temperatures. Journal of Food Engineering, Londres, v.14, n.4, p.269-287, 1991. 